I: :esnted at the 1991 Particle Accelerator Conference, San Fran., Calif., 5/6-9/91

BNL -45463

\title{
TRANSIENT ANALYSIS. OF THE AGS-BOOSTER RING DIPOLE AND QUADRUPOLE MAGNET SYSTEM ${ }^{1}$
}

\author{
W. Zhang, A.V. Soukar. and S.Y. Zhang \\ AGS Department, Brookhaven National Laboratory, Upton, NY 11973 \\ BNL --45463 \\ DE91 013589 \\ dith 3 . 999
}

\begin{abstract}
A case study has been conducted for the quant:tative analysis of the transmission line effects in the Brookhaven AGS Booster ring dipole and quadrupole magnet string. The Booster is a rapid cycling synchrotron $(7.5 \mathrm{~Hz})$ which is excited by multiphase rectifier power supplies. A computer model and a simulation program are developed to study the transient current response of the magnet string due to an applied step voltage. To damp out the staircase noise caused by wave reflection during the current ramp, external resistors will be added in parallel with each half dipole magnet and each quadrupole magnet. The system model and simulation values are based on the actual magnet parameters, the magnet power supply bus sys tem, and the proposed current ramping rate. The system simulation approach can be applied to a larger system as well, and will be briefly discussed.
\end{abstract}

\section{INTRODUCTION}

In the AGS Booster main ring, there are 36 dipoles, 24 horizontal and 24 vertical focusing quadrupoles. The main coils of these magnets are connected in series and powered by multiphase rectifier power supplies. The requirements for the AGS Booster ring power supplies are quite varied. They must aet as accurate low voltage supplies for beam injection; they must ramp rapidly up and down, in two distinctly different modes, for beam acceleration and cycle recovery; and also be capable of flat top operation for a period greater than two seconds for accumulation of polarized proton beams. It is known that magnet strings will behave like a varying impedance transmission lines, due to their distributed inductances and capacitances. This leads to the concern of pulse reflection noise problem during last current ramping. The circuit models of the dipole and quadrupole magnets are shown in Figure 1. In these models, $L_{D}, R_{D}$, $C_{D}$ are the dipole magnet inductance, resistance, or capacitan'e, respectively. And, $L_{Q}, R_{Q}, C_{Q}$ are defined

1 Work performed under the auspices of the U.S. Department of Energy. similarly for the quadrupole magnet. The parameters used in the simulation are summarized in table 1.

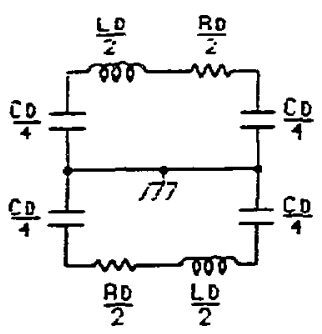

OIPQLE MFGET rooE

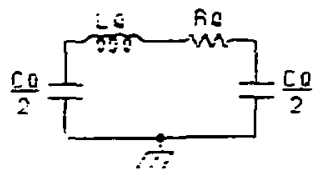

arpquaf Mistet roog
Figure 1. The dipole and quadrupole magnet models.

Table 1. Magnet Parameters

\begin{tabular}{|l|l|l|}
\hline Magnet & Dipole & Ouadrupole \\
\hline Inductance & $3.2 \mathrm{mh}$ & $0.35 \mathrm{mh}$ \\
\hline Resistance & $1.5 \mathrm{mohm}$ & $0.9 \mathrm{mohm}$ \\
\hline Capacitance & $10 \mathrm{nf}$ & $2 \mathrm{nf}$ \\
\hline
\end{tabular}

A simplified schematic of magnet bus connection and power station arrangement is shown in Figure 2.

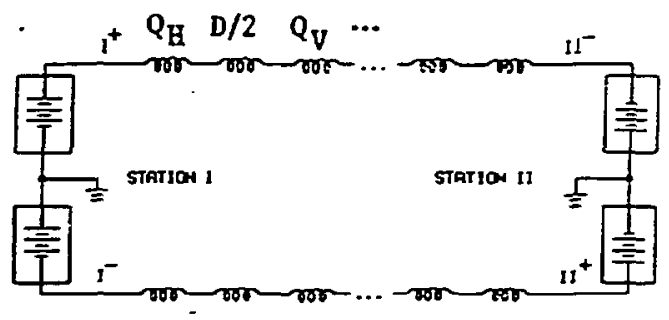

Figure 2. Simplified schematic of the AGS-Booster ring magnet power supoly system.

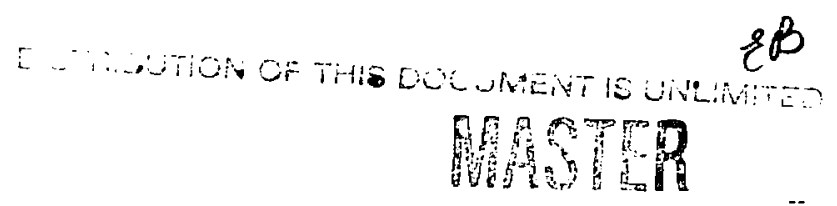


This arrangement virtually splits the circuit into two identical strings. One is from $I^{+}$to $I^{-}$, and another is from $\mathrm{II}^{+}$to $I^{-}$. There are 12 identical sections in each string, a total of 24 sections in the ring. Figure 3 shows a typical section consisting of 2 quadrupole magnets and 3 top or bottom halves of dipole magnets. The computer simulations are based on the circuit model of one entire string powered by two supplies, one at each end, with the same voltage amplitude but opposite polarities.

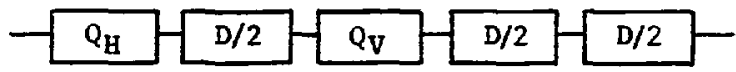

Figure 3. A typical section of the magaet string.

\section{SIMULATION RESULTS}

The transient current response due to an applied voltage step is the main interest in this study. In the AGS Booster operation, the magnet current must ramp up linearly from 600 amperes to 2500 amperes in $60 \mathrm{~ms}$. This requires a voltage step about $+/-1068$ volts at each end.

Each magnet string is a balanced circuit about the center. Therefore, the center point is a virtual short of the transmission line, when the magnet string is powered by a pair of power supplies with equal amplitude and reversed polarity. The wave reflection time is twice the half way delay time of the transmission line. The initial magnitude of the staircase noise is about $U / Z$, where $U$ is the voltage step, and $Z$ is the average impedance of the transmission line.

To damp out the noise, fisst we added an external resistor in parallel with each half dipole magnet. As one might expect, the result shows that the smaller the external resistance, the faster the noise damping rate. However, the current sharing between the external resistor and the dipole magnet results in a current difference between the dipole and quadrupole magnets. For better tracking of the quadrupole and dipole fields in the Booster ring, it is necessary to put an external damping resistor in parallel with each quadrupole magnet as well as across the dipole magnet.

The damping resistor used with each half of the dipole magnet is $300 \Omega$, as suggested by $M$. Meth in [4], and a $50 \Omega$ resistor is used for each quadrupole magnet. Figure 4 shows 6 current curves of quadrupole and dipole magnets located at the first, middle, and last section of the magnet string, for the first $2 \mathrm{~ms}$ after the excitation. Figure 5 (a) shows the absolute current difference, as a percentage, between quadrupole magnets and the design value, and (b) of the dipole magnets. It can be seen that the current differenc reduce to below $0.01 \%$ in $600 \mu \mathrm{s}$. The average abs lute current difference of dipole and quadrupole ma nets to the design value is shown in Figure 6, where decreases to less than $0.01 \%$ in about $200 \mu \mathrm{s}$. It $\mathrm{m}$ : be noticed that this scheme now contains a hi impedance resistive bypass with the main magn string. Therefore, the nonlinearity of current rampi caused by parallel and series resistance should be tak into account. Figure 7 shows the dipole and quadr pole magnet current differences to the design value percent. The peak difference is about $0.76 \%$. A cure this problem is to apply small trimming steps durir current ramping, and current tracking to the comman will not be a problem. Compared to the initial ste each trimming step must be fine enough, so that th reflection noise will not become noticeable.

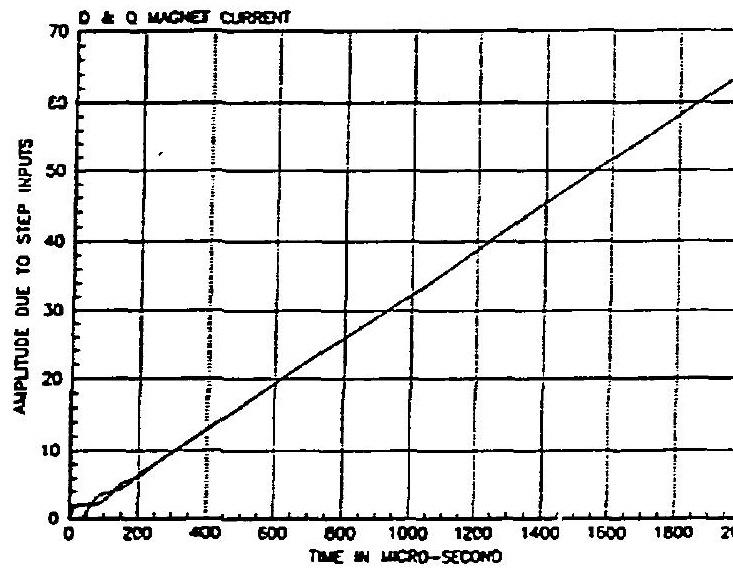

Figure 4 Curreal response of dipole and quadrupole magnets.

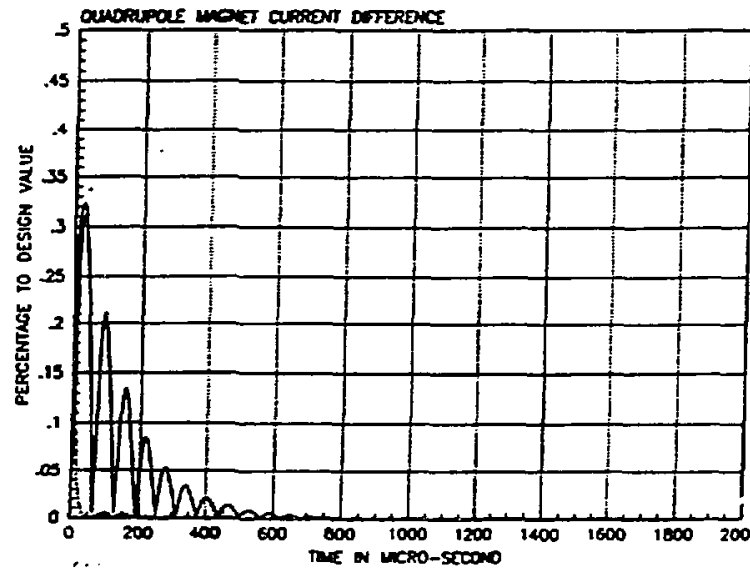

Figure 5 (2). The absolote current difference between gandrupole magnet 


\section{SIMULATION APPROACH}

The circuit model of the AGS Booster ring magnet string is a high order ladder network. For an $N$ section $\mathrm{L}-\mathrm{C}$ ladder network, the network matrix size isusually $2 N \times 2 N$, which can easily go beyond the capability of the electronic simulation programs. To simulate the Booster ring magnet string, we formulaie the network in state space form. The voltage across the capacitor and the current through the inductor are chosen as the state variables. Since most high order ladder network have all the distinctive eigenvalues, the simulation can be obtained by using an eigen-system analysis approach. Applying a similarity transformation to the state equation, the output can be converted to a summation of the exponential functions. In this simulation, time step iteration is not involved. The simulation accuracy depends on the circuit model and eigen-parameter computation. This method turns out to be effective and of better simulation accuracy. However, it is restricted that the network must be a linear system. This is an extension of the method we used to simulate our fast kicker systems. Some detailed information is given in reference [5]. The circuit is simulated in $M A T R D X_{X}{ }^{2}$. We developed our own user code. Expanding the stack size of the program, this method can be applied to a larger system.

\section{ACKNONLEDGEMENT}

The authors wish to thank Mr. Roger Karz and Mr. Richard Casella for their support in using the Apollo system, also Dr. M. Meth for useful disewsions about the problem.

\section{REFERENCES}

[1] "Booster Design Manual," AGS Booster Project, Brookhaven National Laboratory, 1988.

[2] O. Calvo and G. Tol, "Analysis of Transmission Line Effects in the SSC Magnet System," Proc., 1987 PAC.

[3] O. Calvo, et al., Magnet Current Regulation in the SSC," Proc., 1987 PAC.

[4] M. Meth, Magnet Wave Propagation,- AD Booster tech. note \#136, BNL, 1989.

[5] W. Zhang, et al., "A PFN and Transmission Line Simulation Method for Energy Discharge Systems," IEEE Conf. Rec., 19th PMS, 1990.

${ }^{2} M A T R L X_{X}$ is a trademark of Integrated Systems Ior.. Santa Clara, CA 95654 .

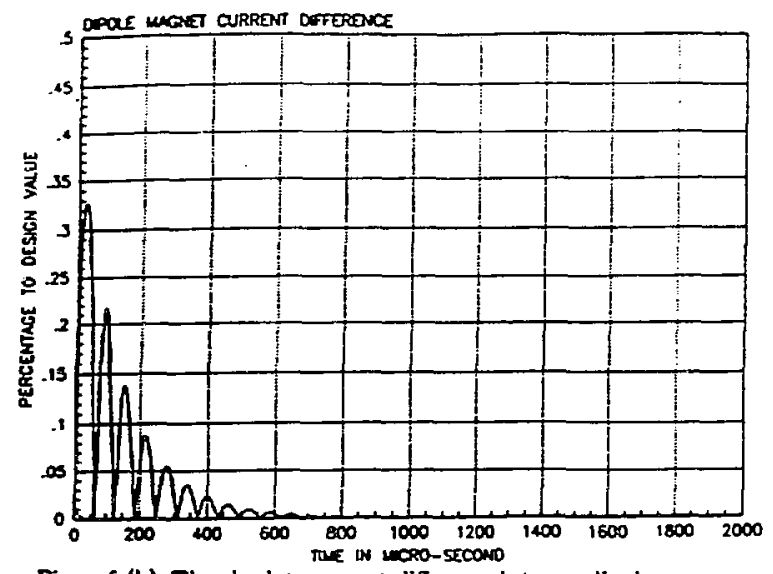

Figare 5 (b) The absolute corrent difference betweed dipole magats.

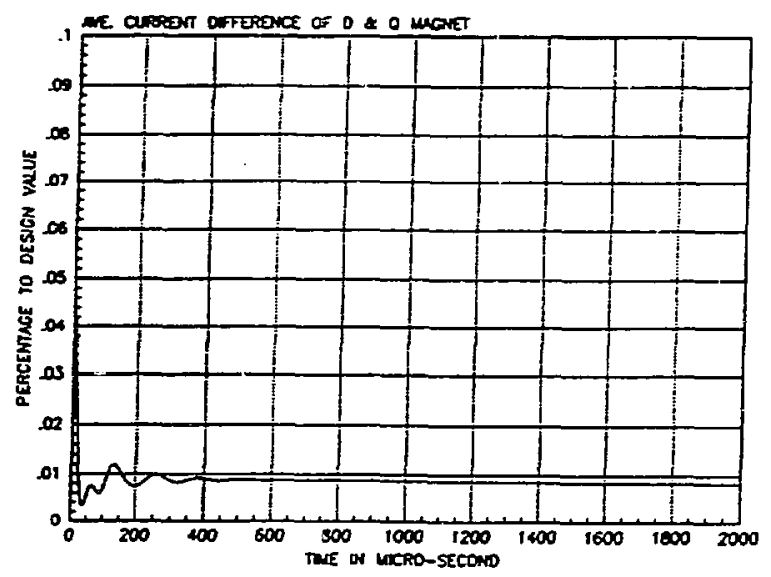

Figure 6. The average absolute curreat diference of quadrupale and dipole magnets.

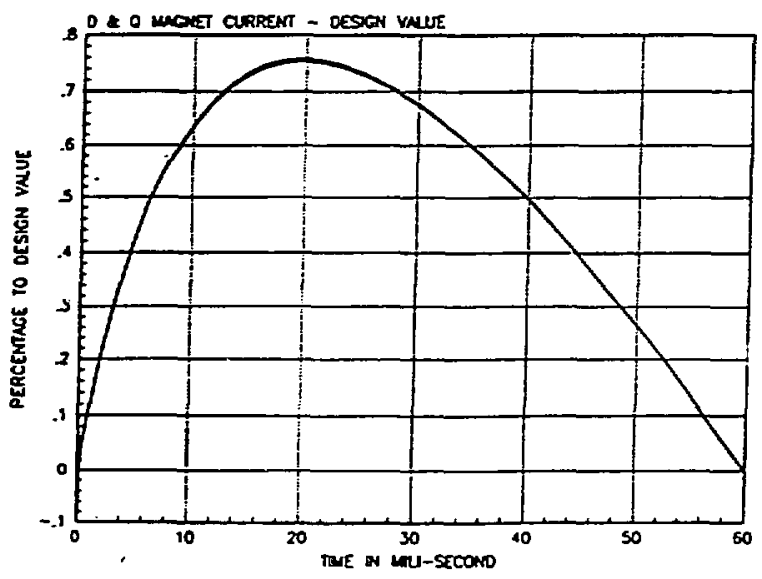

Figure 7. Curreat difference to the design value. 